\title{
Improving Performance of IQ Test for Children with Neurodevelopmental Conditions by Prolonged Exposure to Blue Color
}

\author{
I. Elaraby ${ }^{*} \mathbb{C}^{\circ}$, Ossama A. Abdou ${ }^{2}$ \\ ${ }^{1}$ Helwan University, Cairo, Egypt \\ ${ }^{2}$ Misr International University, Cairo, Egypt \\ Email: ^ib_elhady@yahoo.com
}

How to cite this paper: Elaraby, I., \& Abdou, O. A. (2021). Improving Performance of IQ Test for Children with Neurodevelopmental Conditions by Prolonged Exposure to Blue Color. Psychology, 12, 223235

https://doi.org/10.4236/psych.2021.122014

Received: December 9, 2020

Accepted: February 7, 2021

Published: February 10, 2021

Copyright $\odot 2021$ by author(s) and Scientific Research Publishing Inc. This work is licensed under the Creative Commons Attribution International License (CC BY 4.0).

http://creativecommons.org/licenses/by/4.0/

(c) (i) Open Access

\begin{abstract}
This research paper aims to demonstrate the effect of the blue color on children with neurodevelopmental conditions in particular, in order to improve their specifications. We have shown from previous studies the positive effect of blue color on such participants. So, we tried to confirm this result in this paper. The study included 40 participants with different neurodevelopmental conditions. We exposed the participants to the blue color. Exposing ranged from 3 hours per week for six months to 25 hours per week for the same period. Intelligence tests were administered for each participant, before starting and six months after the first test. The study showed that exposure to blue color led to an increase in the average percentage of participants' intelligence quotient (IQ). The increase doubled with increasing exposure time to blue color. It is expected that the difference in the effect will appear through the intelligence tests which were applied to different participants of children with neurodevelopmental conditions.
\end{abstract}

\section{Keywords}

Color, Bleu, Intelligent, Children, Neurodevelopmental Conditions, Improve, Specifications, Tests, Exposure, Architectural Spaces

\section{Introduction}

Throughout history and up to the present day, color plays an important and major role in human life (Darius, 2011; Elaraby \& Mohsen, 2013). This importance is associated with traditions, customs, ceremonies and psychotherapy (Elaraby \& Aly, 2018; Jung et al., 2018). Color is an essential element in the design, and it has a special importance that does not share or match any other element 
of architectural design, because each element needs color to be recognized (Iyad, 2010; Hammoudah, 1983). Color affects the human psyche, and activates various emotions in their depths, such as sadness, calmness and turmoil (Elaraby \& Aly, 2018). Intellectual disability affects about $2 \%-3 \%$ of the general population (Intellectual Disability, https://en.wikipedia.org/wiki/Intellectual disability) means about 170 million people. One of the reasons and significance of choosing this topic is the fact that many building designers (architects as well as interior designers) may not give enough scientific thoughts and attention when it comes to selecting colors for interior building spaces, especially in neurodevelopmental cases. Since the colors of the spaces affect humans (Gabriel, 2013; Muhammad, 2010), and it has a clear effect in helping patients (Saeed, 2009; Shahenda, 2015; Yassin, 2014). Hence, this study serves as a guide and incentive to recommendations for selecting proper colors for interior spaces, given certain circumstances facing building designers that relate to specific human needs. In the current research, the effect of the blue color on the IQ score of neurodevelopmental conditions was evaluated. Where intelligence is defined as the sum of mental abilities, and the IQ score reflects good performance in intelligence tests (Al-Shafi'i, 2017; Bakri, 2014). A field study was conducted for patients with low and medium IQs. This research assesses the problem of how to help people with mental problems using blue. The aim of the research was to determine the possibility of improving the level of IQ of people with neurodevelopmental conditions.

\section{Experience}

We conducted a study on the effect of the nine colors (white, black and the seven colors of the spectrum) on the children with neurodevelopmental conditions (Elaraby \& Aly, 2018), and the study showed that the blue color had the largest positive effect on children with a fixed 6 degree on all participants of the sample, followed by the green color with an average score of 5.75 degrees. Another study on two groups having similar conditions conducted by us, one group was exposed to blue, and another (control group) was not exposed to blue, where the difference in the average IQ scores between the two groups was 2.2 degrees on the Stanford-Binet scale.

1) We conducted the study on 40 participants that visited a rehabilitation center.

2) The age of each participant was determined at the start of the study.

3) The clinical status of each participant has also been determined.

4) We divided the participants into two groups: the first of 17 participants and the second 23.

5) Blue curtains were used in the rehabilitation center, to have the desired effect.

6) The first group was exposed to blue color for one hour a day, 5 days a week, at a rate of 5 hours per week, equal 25 hours a week.

7) The second group was exposed to blue for one hour a day, 3 days a week, equal 3 hours a week.

Intelligence tests were taken for the two groups at the start of the trial, by psychologists and then the tests were repeated after 6 months. 
8) The difference between IQ scores was calculated for each participant between the pretest and posttest.

\subsection{Participants Data}

Forty different participants were dealt with.

Their ages ranged during a period of 2 years, 3 months, 21 days and 13 years, 3 months, 21 days, with an average age of 5 years, 9 months and 10 days.

The participants' IQ scores at pretests ranged from 40 to 105, with an average score of 70.88 .

Participants clinical status were as the following.

Linguistic delay (8 cases), Simple autism ( 5 cases), Simple mental disability (4 cases), Learning difficulties (4 cases), Simple autism and hyperactivity (2 cases), Autism features ( 2 cases), Moderate autism (2 cases), Simple cerebral palsy (2 cases), Hyperactivity (2 cases), Mental retardation (one case), Severe autism (one case), Down's syndrome (one case), Cerebral palsy (one case), Learning difficulties and problem of attention deficit (one case), Optional silence (one case), Hearing impairment and cochlear implants (one case), Hyperactivity and severe distraction (one case), Electrocardiogram on the brain (one case), and Simple mental syndrome (one case).

\subsection{Statistical Indications}

To confirm the reliability of the results, we performed two statistical tests.

\subsection{1. (T) Test to Reveal the Significance of Statistical Differences} The value of $(t)$ was calculated for the group (IQ scores for the pretest).

where: $(D)$ is the difference between the two degrees, pretest and posttest. $(N)$ is the number of cases.

$$
t=\frac{\sum D}{\frac{N \sum D^{2}-\left(\sum D\right)^{2}}{N-1}}
$$

By calculating the value of $T$ for the group, the result was 0.131 , which is less than the scheduled value at (0.05), which is 2.26 . That is, there are no significant statistical differences in the group.

\subsubsection{Calculation of the Correlation Coefficient}

The correlation coefficient between degrees was calculated before exposure to blue and after exposure (Al-Janabi, 2012). The correlation coefficient was 0.98, which means that the correlation is very strong and indicates the extent of the correlation of the degrees after the increase after exposure to the blue color with the original degrees before exposure to the color.

$$
R=\frac{\sum x * y-\frac{\sum x * \sum y}{n}}{\sqrt{\frac{\left(\sum x^{2}-\left(\sum x\right)^{2}\right)}{n} \frac{\left(\sum y^{2}-\left(\sum y\right)^{2}\right)}{n}}}
$$


whereas:

$X=$ the first variable. $Y=$ the second variable. $N=$ the number of the sample.

\subsection{Exposing Children to Blue Color}

Figure 1 shows four different pictures of the children. The activity room was provided with blue curtains to create the desired effect of the color, so that the results express the color effect (Harald, 2012; Harleman, 2007).

The figure shows a picture of the children while exposed to the blue color (the curtains) in the rehabilitation center.

\subsection{Degrees of Pretest and Posttest Intelligence}

Table 1 shows the group's scores at the start of the experiment, and the scores after period of six months, where we made tests for each participant, and the age of each of them was determined upon the test (pre-test), in order to determine and classify the degree of IQ of each one, and thus compare it with The IQ score resulting from the second test (posttest).

According to Table 1, the sample ages ranged between two years, three months and 21 days, to 13 years, 3 months and 21 days, with an average age of five years, 9 months and 10 days, pretest IQ scores ranged between 40 degrees and 105 degrees, with an average of 70.88 degrees, while the posttest scores ranged between 43 degrees and 105 degrees with an average of 73.45 degrees, and the difference between them ranged between -6 degrees and 11 degrees with an average of 2.58 , which is a good general average that shows the positive effect of the blue color on improving the IQ performance of them, where those who did not exposed to blue recorded -0.2 according from previous study (Elaraby \& Aly, 2020). Figure 2 shows the difference between pretest and posttest degrees.

It is shown from the figure that the posttest IQ scores were more increase between the grades $(40-68)$, where 21 participants recorded increases in the scores, then began to fluctuate between the degrees (69-105), eight of them recorded increases while 5 ones recorded (zero) and 6 cases recorded negative values.

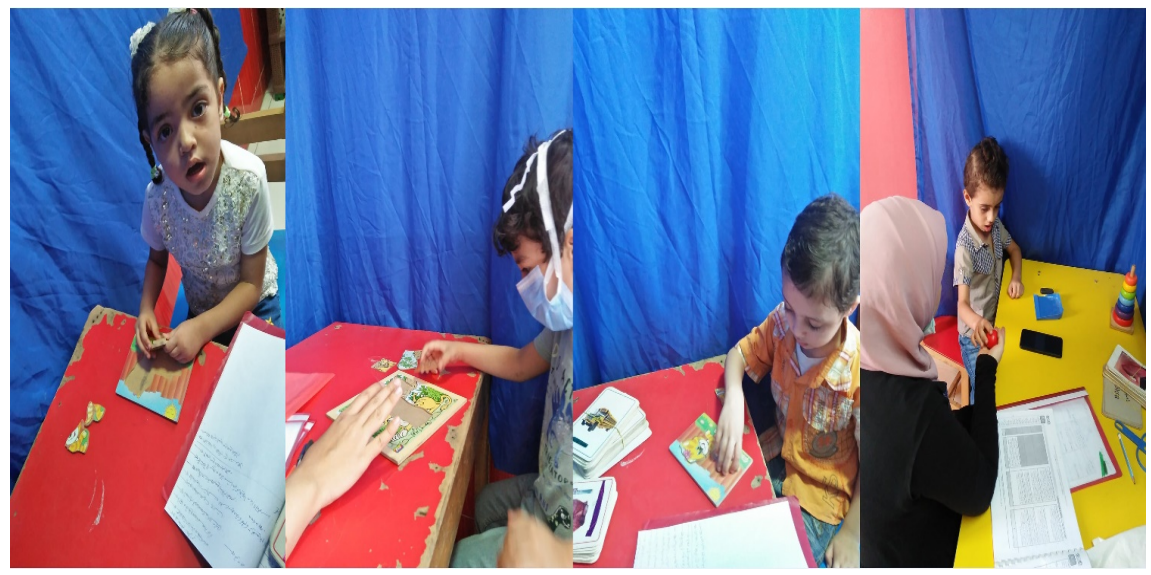

Figure 1. Pictures of children while they were in the skills development center. 


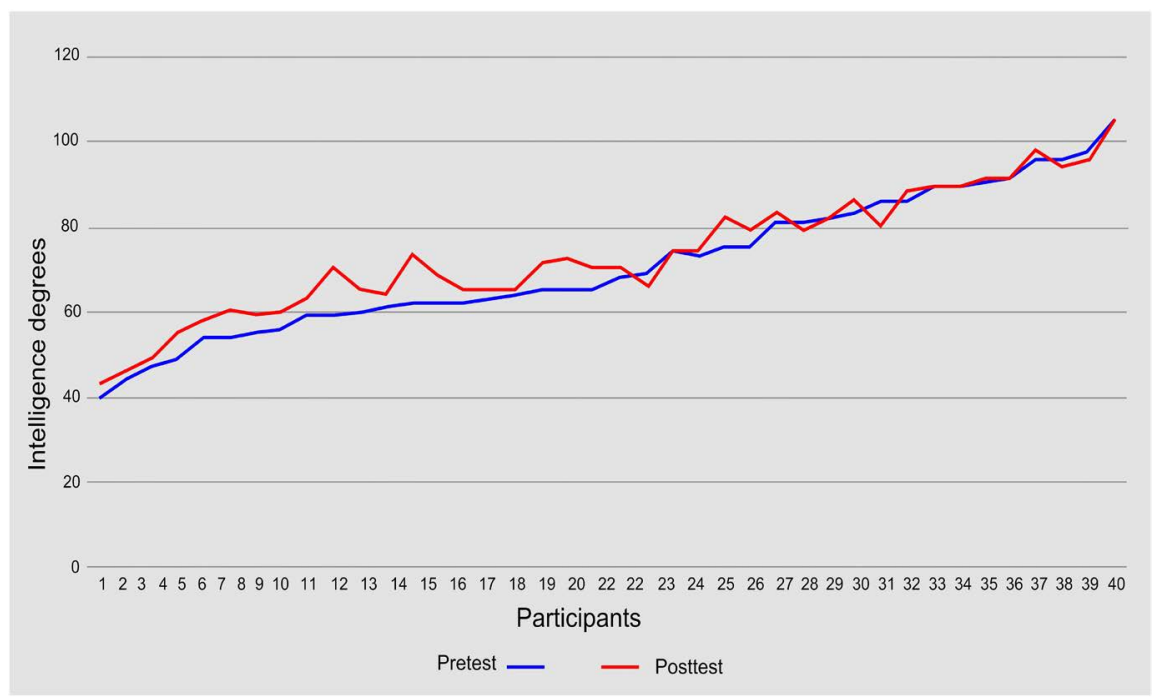

Figure 2. The difference between pretest and posttest IQ degrees.

Table 1. Pretest and posttest scores, classification and diagnosis of participants.

\begin{tabular}{|c|c|c|c|c|c|c|c|}
\hline No. & Gender & Age & Pre-test & Post test & Difference & Clinical diagnosis & Classification \\
\hline 1 & female & 12.59 & 63 & 65 & 2 & Simple mental disability & Late or slightly weakness \\
\hline 2 & female & 2.95 & 73 & 74 & 1 & Delayed language & Late or Interstitial weakness \\
\hline 3 & female & 2.56 & 75 & 79 & 4 & Autism features & Late or Interstitial weakness \\
\hline 4 & male & 7.01 & 54 & 60 & 6 & Mental retardation & Late or slightly weakness \\
\hline 5 & male & 9.28 & 62 & 65 & 3 & Learning difficulties & Late or slightly weakness \\
\hline 6 & male & 5.37 & 56 & 60 & 4 & Difficulties learning and poor attention & Late or slightly weakness \\
\hline 7 & male & 10.56 & 62 & 68 & 6 & Hearing impairment and cochlear implantation & Late or slightly weakness \\
\hline 8 & male & 4.8 & 86 & 80 & -6 & Simple mental syndrome & Below average \\
\hline 9 & male & 4.07 & 82 & 82 & 0 & Delayed language & Below average \\
\hline 10 & male & 4.1 & 65 & 70 & 5 & Simple autism & Late or slightly weakness \\
\hline 11 & male & 4.8 & 44 & 46 & 2 & brain paralysis & Late or medium weakness \\
\hline 12 & male & 5.19 & 54 & 58 & 4 & Simple autism & Late or medium weakness \\
\hline 13 & male & 3.93 & 90 & 89 & -1 & Delayed language & Average \\
\hline 14 & male & 3.87 & 65 & 72 & 7 & Simple autism & Late or slightly weak \\
\hline 15 & male & 4.49 & 92 & 92 & 0 & Delayed language & Average \\
\hline 16 & female & 5.96 & 61 & 64 & 3 & Simple mental disability & Late or slightly weak \\
\hline 17 & female & 4.81 & 89 & 89 & 0 & Delayed language & Below average \\
\hline 18 & male & 3.33 & 68 & 70 & 2 & Simple autism & Late or slightly weak \\
\hline 19 & female & 5.54 & 81 & 83 & 2 & Delayed language & Below average \\
\hline 20 & male & 4.9 & 96 & 98 & 2 & Simple autism and hyperactivity & Average \\
\hline 21 & female & 6.31 & 73 & 74 & 1 & Simple brain paralysis & Late or Interstitial weakness \\
\hline 22 & male & 6.91 & 98 & 96 & -2 & Learning difficulties & Average \\
\hline 23 & female & 6.31 & 49 & 55 & 6 & Medium autism & Late or medium weakness \\
\hline
\end{tabular}




\section{Continued}

\begin{tabular}{|c|c|c|c|c|c|c|c|}
\hline 24 & male & 6.6 & 59 & 63 & 4 & Hyperactivity and distraction & Late or slightly weakness \\
\hline 25 & male & 13.31 & 55 & 59 & 4 & Simple brain paralysis & Late or slightly weakness \\
\hline 26 & male & 8.89 & 81 & 79 & -2 & Hyperactivity & Below average \\
\hline 27 & male & 4.02 & 62 & 73 & 11 & Autism features & Late or slightly weakness \\
\hline 28 & male & 5.98 & 83 & 86 & 3 & Learning difficulties & Below average \\
\hline 29 & male & 3.76 & 96 & 94 & -2 & Delayed language & Average \\
\hline 30 & male & 5.54 & 47 & 49 & 2 & Down syndrome & Late or medium weakness \\
\hline 31 & male & 5.92 & 69 & 66 & -3 & Hyperactivity & Late or slightly weakness \\
\hline 32 & male & 4.24 & 105 & 105 & 0 & Learning difficulties & Average \\
\hline 33 & male & 9.45 & 40 & 43 & 3 & Severe autism & Late or medium weakness \\
\hline 34 & female & 3.4 & 60 & 65 & 5 & Electricity on the brain & Late or medium weakness \\
\hline 35 & female & 3.79 & 64 & 65 & 1 & Optional silence & Late or Interstitial weakness \\
\hline 36 & female & 6.2 & 91 & 91 & 0 & Simple mental disability & Average \\
\hline 37 & female & 3.03 & 59 & 70 & 11 & Simple autism & Late or slightly weakness \\
\hline 38 & male & 2.31 & 75 & 82 & 7 & Delayed language & Late or slightly weakness \\
\hline 39 & male & 5.98 & 86 & 88 & 2 & Simple autism & Below average \\
\hline 40 & male & 9.03 & 65 & 71 & 6 & Simple mental disability & Late or slightly weakness \\
\hline \multicolumn{2}{|c|}{ Average } & 5.78 & 70.88 & 73.45 & 2.58 & & \\
\hline \multicolumn{2}{|c|}{ St. deviation } & 2.57 & 16.34 & 14.69 & 3.49 & & \\
\hline
\end{tabular}

\subsection{The Difference in the Increase between Males and Females}

Table 2 and Table 3 show the increases in IQ scores for both males and females.

Figure 3 shows the average difference in the increase in IQ scores between males and females according to results of pretest and posttest.

The tables and the figure show that the average increase in IQ scores for females was slightly higher than the average increase for males. The average increase for females was 3.16 degrees, while the average increase for males was 2.39 , and this increase is a kind of convergence between the average scores for the two sexes, without much preference for one over the other.

\subsection{The Relationship of Age to the Increase in IQ Scores}

Figure 4 shows the relationship between age and the increase in IQ scores for all participants.

Age of each participant was determined when doing the first test (pretest), and then we studied the relationship of age to the change in IQ, compared to the second test (the posttest), and we got the following results. The number of participants whose IQs increased were 29 Increase by $72.5 \%$. The number that their IQs did not change were 5 , by $12.5 \%$. Finally, the number whose IQs decreased were 6 by 15\%. The improvement was not limited to an increase in IQ scores, but there was a noticeable improvement in the two cases that obtained 11 points 
increase, one of which was due to improvement in the social aspect, while the improvement in the other was in the linguistic side. The figure shows that the increase in IQ scores was mostly greater with youngers, and less with older, which means that younger are more responsive and benefit from older.

Table 2. The difference in the increase of IQ scores females.

\begin{tabular}{|c|c|c|c|}
\hline No. & Pretest & Posttest & difference \\
\hline 1 & 65 & 71 & 6 \\
\hline 2 & 86 & 88 & 2 \\
\hline 3 & 75 & 82 & 7 \\
\hline 4 & 40 & 43 & 3 \\
\hline 5 & 105 & 105 & 0 \\
\hline 6 & 69 & 66 & -3 \\
\hline 7 & 47 & 49 & 2 \\
\hline 8 & 96 & 94 & -2 \\
\hline 9 & 83 & 86 & 3 \\
\hline 10 & 62 & 73 & 11 \\
\hline 11 & 81 & 79 & -2 \\
\hline 12 & 55 & 59 & 4 \\
\hline 13 & 59 & 63 & 4 \\
\hline 14 & 98 & 96 & -2 \\
\hline 15 & 96 & 98 & 2 \\
\hline 16 & 68 & 70 & 2 \\
\hline 17 & 92 & 92 & 0 \\
\hline 18 & 65 & 72 & 7 \\
\hline 19 & 90 & 89 & -1 \\
\hline 20 & 54 & 58 & 4 \\
\hline 21 & 44 & 46 & 2 \\
\hline 22 & 65 & 70 & 5 \\
\hline 23 & 82 & 82 & 0 \\
\hline 24 & 86 & 80 & -6 \\
\hline 25 & 62 & 68 & 6 \\
\hline 26 & 56 & 60 & 4 \\
\hline 27 & 62 & 65 & 3 \\
\hline 28 & 54 & 60 & 6 \\
\hline Average & 71.32 & 73.71 & 2.39 \\
\hline St. deviation & 17.85 & 16.20 & 3.65 \\
\hline
\end{tabular}


Table 3. The difference in the increase of IQ scores for males.

\begin{tabular}{cccc}
\hline No. & Pretest & Posttest & difference \\
\hline 1 & 59 & 70 & 11 \\
2 & 91 & 91 & 0 \\
3 & 64 & 65 & 10 \\
4 & 60 & 65 & 9 \\
5 & 49 & 55 & 8 \\
6 & 73 & 74 & 7 \\
7 & 81 & 83 & 6 \\
8 & 89 & 89 & 5 \\
9 & 61 & 64 & 82 \\
10 & 75 & 79 & 4 \\
11 & 73 & 74 & 3 \\
12 & 63 & 65 & 2 \\
Average & 69.83 & 72.83 & 3 \\
St. deviation & 12.77 & 10.97 & 3.16 \\
\hline
\end{tabular}

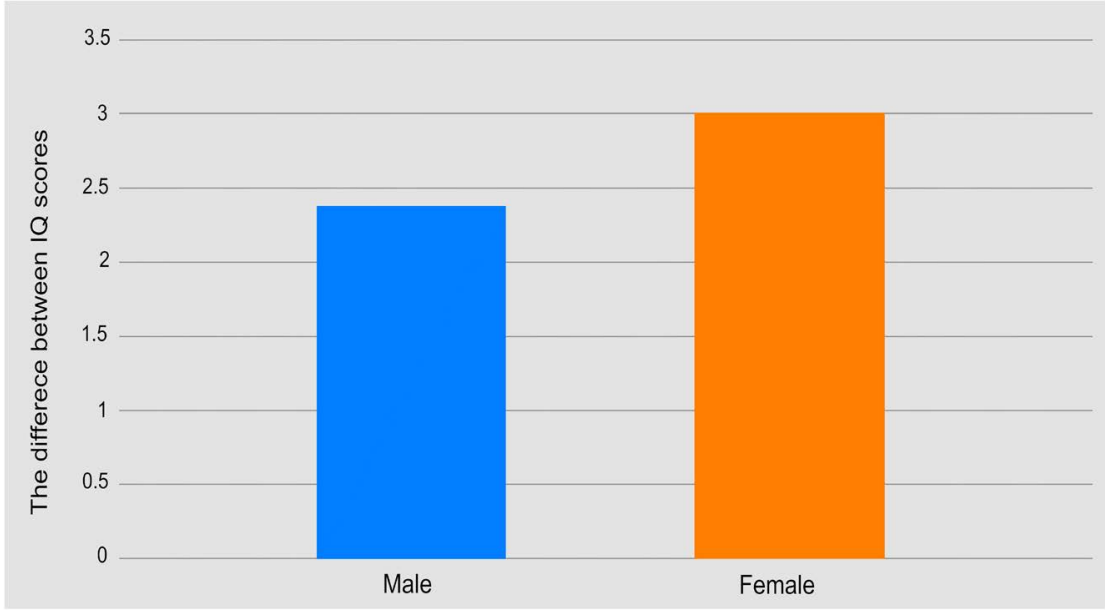

Figure 3. The average difference in the increase in IQ scores between males and females.

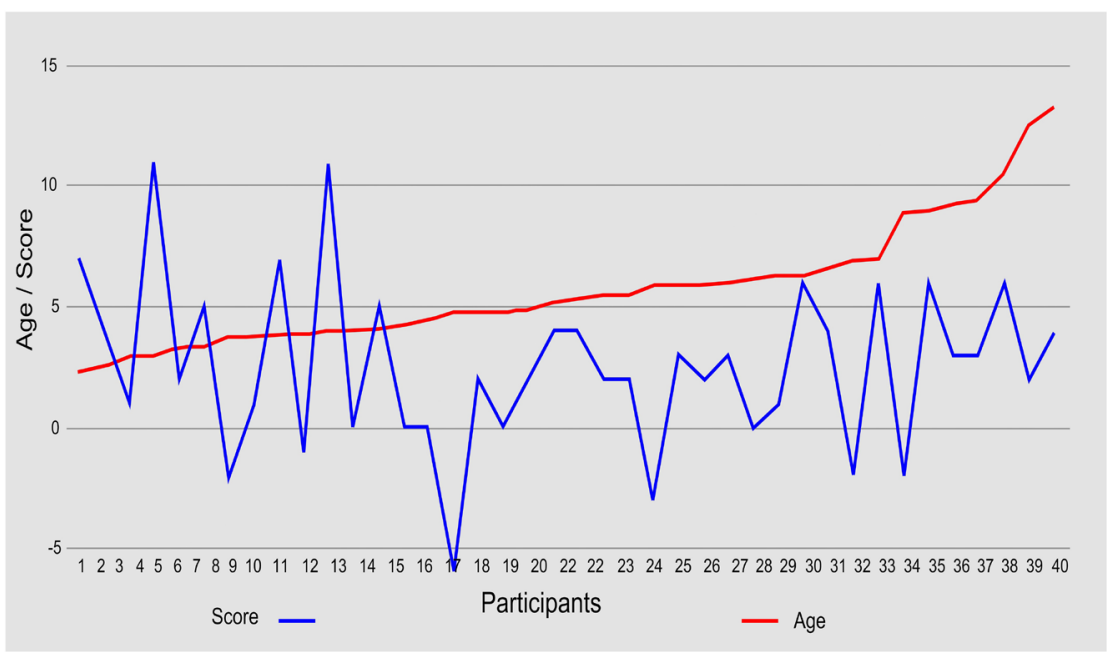

Figure 4. The relationship between age and increase in IQ scores. 


\subsection{The Relationship of the Clinical Case to the Increase in IQ Scores}

Table 4 shows the average increase in IQ scores for each clinical case, while Figure 5 shows the average increase in IQ scores for each case.

Figure 5 shows average IQ scores for each clinical diagnosis. High scores with green color, medium and low with cyan, and minus with red.

The table and the figure show that the number of participants in each case ranged between 1 and 8 participants, and the average increase for each diagnosis ranged between -6.5 and 7.5 degrees, where the negative degrees were for simple mental syndrome and hyperactivity, while the rest of diagnosis had positive average increases. Chief of them are autism features, mental retardation, (Hearing impairment and cochlear implants), simple autism and moderate autism.

Table 4. The averages of the increase in IQ scores for each clinical diagnosis.

\begin{tabular}{|c|c|c|c|}
\hline No. & Clinical diagnosis & $\begin{array}{l}\text { No. of } \\
\text { cases }\end{array}$ & $\begin{array}{c}\text { Mean of } \\
\text { increasing }\end{array}$ \\
\hline 1 & Simple mental disability & 4 & 2.75 \\
\hline 2 & Linguistic delay & 8 & 0.88 \\
\hline 3 & Mental retardation & 1 & 6 \\
\hline 4 & Simple autism & 5 & 5.4 \\
\hline 5 & Simple autism and hyperactivity & 2 & 2 \\
\hline 6 & Severe autism & 1 & 3 \\
\hline 7 & Moderate autism & 2 & 5 \\
\hline 8 & Down's syndrome & 1 & 2 \\
\hline 9 & Autism features & 2 & 7.5 \\
\hline 10 & Cerebral palsy & 1 & 2 \\
\hline 11 & Simple cerebral palsy & 2 & 2.5 \\
\hline 12 & Learning difficulties & 4 & 1 \\
\hline 13 & Learning difficulties and problem of attention deficit & 1 & 4 \\
\hline 14 & Optional silence & 1 & 1 \\
\hline 15 & Hearing impairment and cochlear implants & 1 & 6 \\
\hline 16 & Hyperactivity & 2 & -2.5 \\
\hline 17 & Hyperactivity and severe distraction & 1 & 4 \\
\hline 18 & Electrocardiogram on the brain & 1 & 5 \\
\hline 19 & Simple mental syndrome & 1 & -6 \\
\hline Average & & 2.16 & 2.45 \\
\hline St. deviation & & 1.86 & 3.08 \\
\hline
\end{tabular}




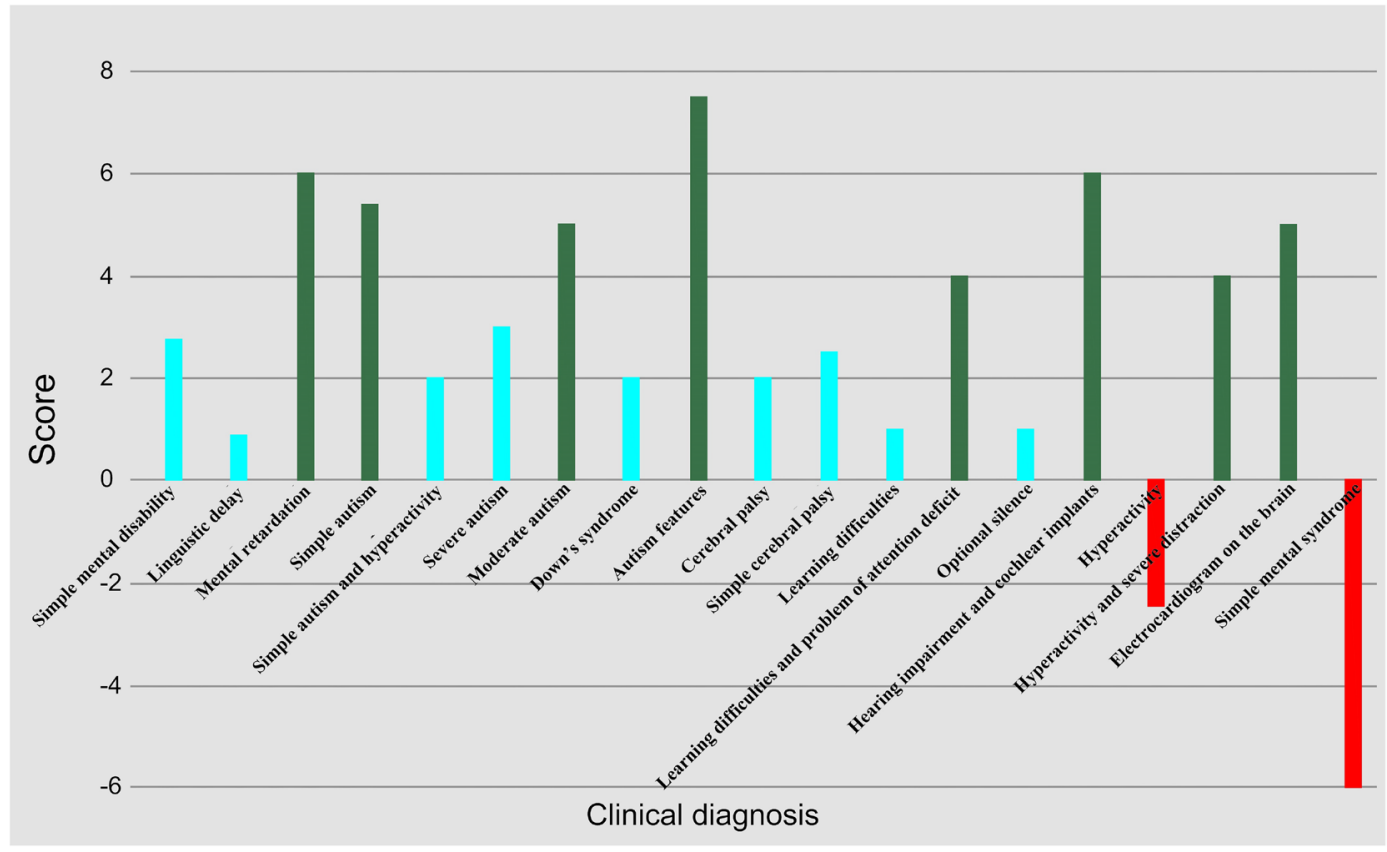

Figure 5. Average IQ scores for each clinical diagnosis.

\subsection{The Relationship of Increased Exposure to Blue Color Increased IQ Scores}

Figure 6 shows the pretest and posttest scores for participants who were exposed to blue for 3 hours per week.

Figure 7 shows the pretest and posttest scores for participants who were exposed to blue for 25 hours per week.

It is shown from Figure 6 that the increases in IQ scores for the group that was exposed to the blue color for 3 hours per week ranged between -6 and 7 degrees, with an average of 1.65 degrees. The number of negative degrees were 6 , while the number of cases in which the increase was zero were 3 , and the number of cases with positive increases were 14 .

It is also shown from Figure 7 that the increases in IQ scores for the group that was exposed to the blue color for 25 hours per week ranged between 0 and 11 degrees, with an average of 3.82. The number of cases in which the increase was zero were two and the number of cases with a positive increase were 15 cases. There were no negative differences.

The effect of increasing the duration of exposure to blue color on increasing IQ scores is very clear due to prolonged exposure to blue.

\subsection{Sensible Impact}

Two of the participants obtained an increase in IQ by 11 degrees, and it was found that these two cases showed tangible progress. One of them showed progress in social response, while the other showed progress in verbal skill. This tangible progress confirms that the increase in IQ scores is not just numbers, but it Affects children abilities significantly. 


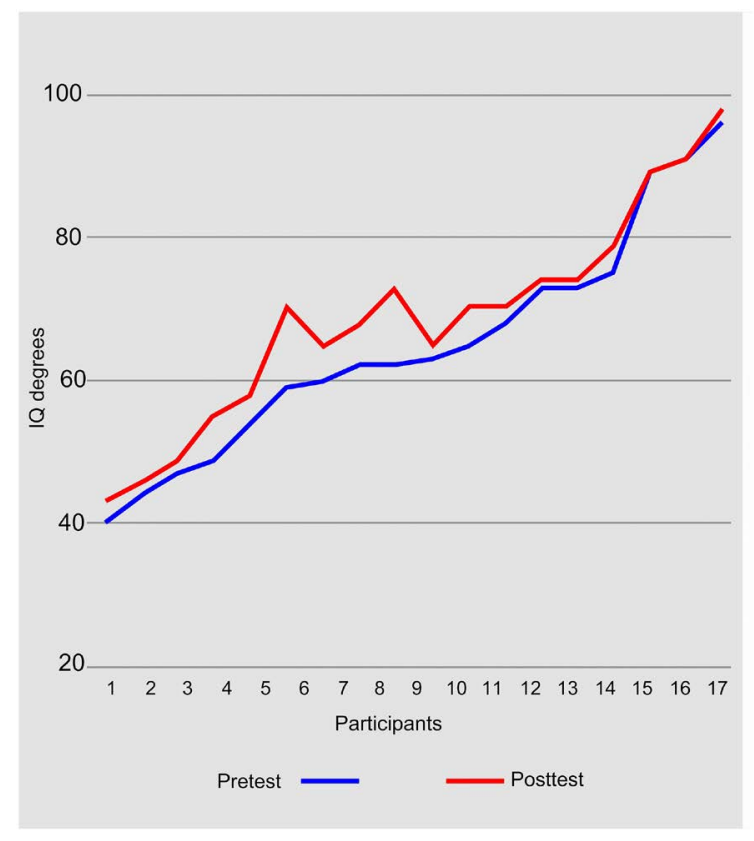

Figure 6. Degrees for who were exposed 25 hours per week.

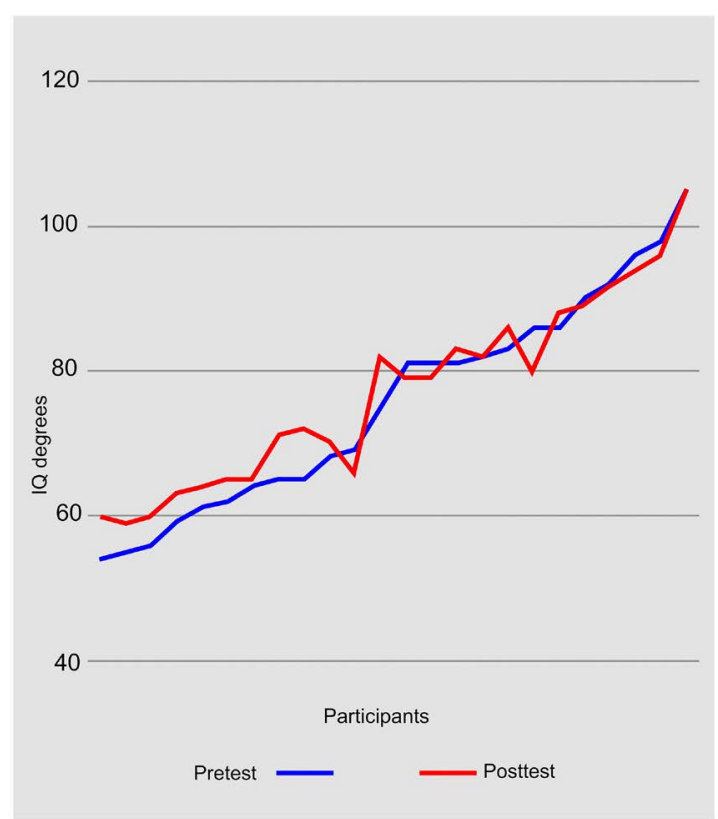

Figure 7. Degrees for who were exposed 3 hours per week.

\section{Conclusion}

The study was conducted on a group of children with neurodevelopmental conditions, who had IQs ranging between 40 and 105 degrees. They were exposed to blue by using blue curtains, for an hour a day at a rate of three days a week to 23 cases, and for 5 Hours a day at a rate of 5 days a week for 17 cases. IQ scores were measured for all cases at the start of the experiment, then 6 months later. The number of participants whose IQs increased were 29 cases by $72.5 \%$. The number that their IQs did not change was 5 , by $12.5 \%$. Finally, the number whose 
IQs decreased were 6 by $15 \%$. The average increase in IQs for all participants was 2.58 degrees, where the average increase for whom exposed for 3 hours a week was 1.65, while the average increase in cases exposed for 25 hours a week was 3.82, equal 2.32 of those were exposed for 3 hours a week. This result confirms that the increase in the period of exposure to blue is directly proportional to the increase of IQ scores. Given the interval between pretest and posttest (six months), it is expected that the child can improve from the level of IQ (40) to the level of an IQ (70) within an approximately period of less than four years.

\section{Recommendations}

1) For future studies

- Continuing studies in the same direction to help these children improving their performance.

- For individual cases, a color test can be done for each case before starting the application.

- Conducting studies on each clinical case, if possible.

2) For interior architects and designers

- Paying attention to the colors of architectural spaces because of their effect on users.

3) For children parents and teachers

- Paying attention for helping youngers, whom seem to be most responsive.

\section{Conflicts of Interest}

The authors declare no conflicts of interest regarding the publication of this paper.

\section{References}

Al-Janabi, S. A. S. (2012). Iraq: Graduate Studies Department, College of Physical Education, Dhi Qar University.

Al-Shafi'i, E. (2017). Intellectual Disability in Children and the Reasons for Their Occurrence. The Arab Republic of Egypt.

Bakri, S. (2014). Stanford-Binet Short Images, Arab Republic of Egypt: The World of Books.

Darius, A. (2011). Color Inspiration. Michigan, USA: North Light.

Elaraby, I., \& Aly, R. (2018). Power of Color. Saarbrücker, Latvia: LAMBERT, Academic Publishing.

Elaraby, I., \& Aly, R. (2020). Study of the Effect of Colors Change on Children with Mental Problems. Journal of Engineering and Applied Science, 67, 411-426.

Elaraby, I., \& Mohsen, R. (2013). Natural Colors and Its Connection to Humans. AIC Conference, New Castle, UK, October 2013.

Gabriel, T. A. R. T. (2013). The Effect of Color in Interior Spaces on the Shopping Activity of Commercial Centers. Master's Thesis, Gaza: Faculty of Engineering, Islamic University.

Hammoudah, Y. (1983). Theory of Color. Cairo: Dar Elmaaref Publishing Company.

Harald, A. (2012). Color and Light Concepts and Confusions. Interim Meeting of the In- 
ternational Colour Association (AIC), Taipei, 22-25 September 2012, 518-521.

Harleman, M. (2007). Daylight Influence on Color Design. PhD Thesis, Stockholm, Sweden: Royal Institute of Technology.

Intellectual Disability. Wikipedia. https://en.wikipedia.org/wiki/Intellectual disability

Iyad, M. (2010). Color Philosophy. Amman: Al Ahlia Publishing and Distribution.

Jung, I., Elaraby, I., Griber, Y., Hanenburg, J., Arbab, Sh., Yoshimurae, K., Ulusoy, B., Johansson, S., \& Samir, E. (2018). Color Associations in Different Cultures. AIC Conference, Lisbon, Portugal, September 2018, 5.

Muhammad, G. J. (2010). Classroom Color Decisions. Iraqi Journal of Architecture, 6, 3.

Saeed, N. O. M. (2009). The Use of Color in a Child's Void. Master's Thesis, Khartoum State, Sudan: Faculty of Fine Arts, and Applied Sciences, Sudan University of Science and Technology.

Shahenda, M., Magda, H., \& Nagwa, H. (2015). Designing for Attention Deficit Hyperactivity Disorder. Master's Thesis, Cairo: Cairo University.

Yassin, R. M. (2014). The Effect of Color Perception on the Recovery Process. Master's Thesis, Damascus: College of Education, University of Damascus. 